\title{
Ovarian cysts in Pregnancy: Obstetric Outcome and Management
}

\author{
Greeshma Singham $^{1}$, Achanta Vivekanand ${ }^{2}$, Satyaprabha Siripurapu ${ }^{2}$, Ipsita Mohapatra ${ }^{2}$
}

${ }^{1}$ Assistant Professor, Department of Obstetrics and Gynecology, ${ }^{2}$ Professor, Department of Obstetrics and Gynecology, Prathima Institute of Medical Sciences, Karimnagar, Telangana, India.

Corresponding author: Greeshma Singham, Department of Obstetrics and Gynecology, Prathima Institute of Medical Sciences, Nagunur, Karimnagar.

Email: greeshma.ch@gmail.com

DOI:10.47799/pimr.0901.14

Date of receiving:6-02-2021

Date of peer review : 15-03-2021

Date of acceptance: 5-04-2021

\section{ABSTRACT}

Objective: To study the pregnancy outcome and management of ovarian cysts during pregnancy.

Methods : A prospective observational study was conducted in Prathima Institute of Medical Sciences, Karimnagar from June 2018 to June 2020. A total of 32 cases with ovarian cysts $\geqslant 5 \mathrm{~cm}$ with benign features as suggested by ultrasound were included in the study. The pregnancy outcome and management were studied.

Results : Out of 32 pregnant women with ovarian cysts, $25(78.12 \%)$ were asymptomatic and were managed conservatively. Of the 25 , spontaneous resolution was seen in $18(56.25 \%)$ patients while $7(21.87 \%)$ patients in whom ovarian cyst persisted or was incidentally detected during cesarean section were managed by cystectomy at the same time. Surgery was needed in the antenatal period in $7(21.87 \%)$ patients due to complications such as increase in size of the cyst seen in $4(12.5 \%)$ and torsion seen in $3(9.37 \%)$. All the 32 patients had good perinatal outcome. There were no miscarriages. All patients continued to term except one, who presented at 36 weeks with torsion, andunderwent emergency cesarean section with oophorectomy. Histopathological examination of the excised cysts showed 5 as simple cyst, 5 as serous cystadenomas, 3 as mucinous cystadenomas and 1 as dermoid.

Conclusion: Management of the ovarian cysts during pregnancy is conservative. Most of them undergo spontaneous resolution. Surgical management is reserved for only symptomatic patients. Pregnancy outcome as such is not affected by ovarian cysts during pregnancy.

Keywords:

Ovarian cysts, Pregnancy.

\section{Introduction}

Ovarian masses diagnosed during pregnancy are of a great concern to the treating doctor and patient. The overall incidence of ovarian masses diagnosed during pregnancy is $0.5-1 \%{ }^{1}$ Majority of them are asymptomatic and undergo spontaneous resolution. ${ }^{2}$ A few maybe malignant requiring more extensive surveillance and surgical management leading to pregnancy loss. The reported incidence of ovarian cancer in pregnant women varies from 0.004-0.04\%. ${ }^{3}$

Most common ovarian masses seen during pregnancy are functional cysts due to hormonal fluctuations such as simple follicular cysts, corpus luteal cysts, hemorrhagic cysts, theca luteal cysts, luteoma of pregnancy. Other benign ovarian masses diagnosed during pregnancy include mature cystic teratomas, serous cystadenomas, mucinous cysts and endometriomas. ${ }^{2,4}$ Malignant ovarian tumor during pregnancy is generally a germ cell tumor or borderline epithelial ovarian tumor. ${ }^{4}$

Most of the ovarian cysts during pregnancy undergo spontaneous resolution. ${ }^{5,6}$ Complications of these cysts during pregnancy include infection, intracystic hemorrhage, rupture of the cyst, torsion, malignant changes, impaction of the cyst in pelvis causing retention of urine, obstructed labour and malpresentation of the fetus.

Surgery is indicated only when a pregnant woman with an ovarian mass presents with acute abdomen due to torsion or rupture or when the mass is suspicious for malignancy, rapidly growing masses, cysts greater than $10 \mathrm{~cm}$ which may cause obstruction of labour. ${ }^{4}$ Torsion of the ovarian cysts is the most common complication during pregnancy presenting with acute abdomen to the emergency department. ${ }^{6}$ Surgical intervention maybe associated with adverse effect on pregnancy such as miscarriage, and preterm delivery. Surgical intervention in second trimester is associated with less adverse 
effects of pregnancy outcome. If ovarian cysts persist throughout the pregnancy, they can be followed up in the postnatal period and managed accordingly. About $0.3 \%$ of the cases of ovarian cysts are incidentally found during cesarean section and can be managed by cystectomy or opherectomy. ${ }^{7}$

\section{Materials and Methods}

The present study was carried out in the Department of Obstetrics and Gynecology, in Prathima Institute of Medical Sciences. It was a prospective and observational study which extended from June 2018 to June 2020. All pregnant women with ovarian cysts $\geqslant 5 \mathrm{~cm}$ and with ultrasonographically benign features were included in our study. A total of 32 patients diagnosed with ovarian cysts by routine ultrasonography or incidentally during delivery or presenting to the emergency department were included in our study. Pregnant women with ovarian cysts suspicious of malignancy based on ultrasonographic features such as presence of solid components, excrescences, papillary structures, internal septations, irregular borders, increased vascularity, low resistance blood flow, ascites were excluded from our study as a multidisciplinary approach preferably at a higher center with specialized experience is necessary to treat these patients and may need termination of pregnancy. Patient demographics, characteristic ultrasound features of the cyst, pregnancy complications arising due to ovarian cyst and management were evaluated. Pregnancy outcome in the form of miscarriage, preterm delivery, mode of delivery were noted. If the patient underwent surgery, specimen was sent for histopathological examination. The collected data was tabulated on windows based personal computer using Microsoft Excel software, and data analyzed using IBM SPSS Statistics (Version 22) software. The data was expressed as mean and percentages.

\section{Results}

There were a total of 52 pregnant women with ovarian cysts. Incidence of ovarian cysts in pregnant women in our study was $0.89 \%$. Among them 32 patients with ovarian cysts $\geqslant 5 \mathrm{~cm}$ and ultrasonographically benign in nature were included in our study. 19 pregnant women who had ovarian cysts $<5 \mathrm{~cm}$ and one woman with malignant features on ultrasound were excluded from our study.

When demographic characteristics were taken into consideration, the mean age of presentation was 25.18 years, $15(46.87 \%)$ belonged to age group $20-24,14(43.75 \%)$ were in the age group $25-29$ and $3(9.37 \%)$ were in the age group 30 34 years. Out of 32 women, $13(40.62 \%)$ were primi gravida and $19(59.37 \%)$ were multigravida. A total of $15(46.87 \%)$ belonged to lower socioeconomic status. At the time of presentation $18(56.25 \%)$ were in first trimester, $9(28.12 \%)$ were in second trimester, $5(15.62 \%)$ in third trimester. Mean gestational age at presentation was 15.96 weeks.(Table 1) Figure 1, Figure 2
In our study 25(78.12\%) patients with ovarian cysts were managed conservatively with mean cyst size $7.6 \mathrm{~cm}$. Of the 25 , $18(56.25 \%)$ patients had spontaneous resolution of the cyst and $7(21.87 \%)$ patients in whom the cyst persisted throughout pregnancy or incidentally found during cesarean, underwent cystectomy at the same time.Out of the 32 patients, surgery was needed in the antenatal period in $7(21.87 \%)$. Of these 7 patients, $4(12.5 \%)$ underwent elective cystectomy in second trimester due to increasing size of the cyst with mean cyst size $19.7 \mathrm{~cm}, 3(9.37 \%)$ patients landed in emergency due to torsion of ovarian cyst with mean cyst size $12.6 \mathrm{~cm}$. Of these 3 patients with torsion, one presented at 9 weeks and underwent laparoscopic cystectomy, another presented at 14 weeks and underwent laparotomy and oophorectomy. Last patient presented at 36weeks with acute abdomen due to torsion, underwent emergency cesarean section along with oophorectomy. (Table 2) Figure 3

In our study no patient had miscarriage. All had a term delivery except one patient who presented with acute abdomen due to torsion at 36 weeks and underwent oophorectomy along with emergency cesarean section. Fourteen(43.75\%) had vaginal delivery and $18(56.25 \%)$ had cesarean section. All the 14 patients who underwent cystectomy as an emergency or elective or during routine cesarean section were sent for histopathological examination. Simple cyst were seen in 5 patients, serous cystadenoma in 5 , mucinous cystadenoma in 3 and dermoid in 1. (Table 3)

Figure 1: Pregnancy with 9 wks gestation with right ovarian cyst of $75 \times 55 \mathrm{~mm}$.

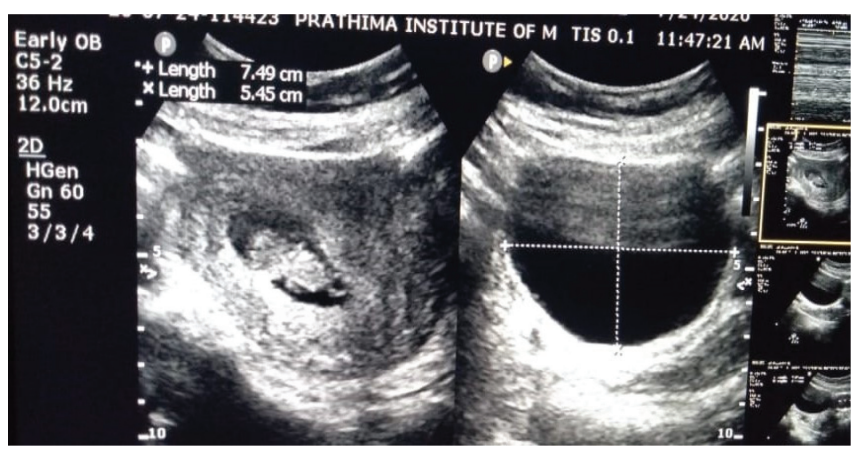

Figure 2: Ovarian cyst Exteriorized after extraction of baby and closure of uterus

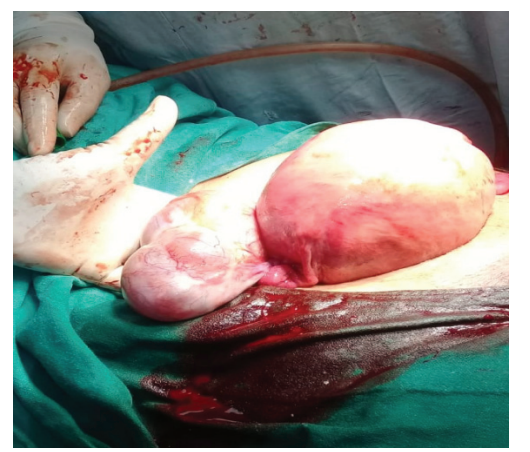


Figure 3: Cesarean section at 36 weeks gestational age with torsion left ovary, presenting as acute abdomen.

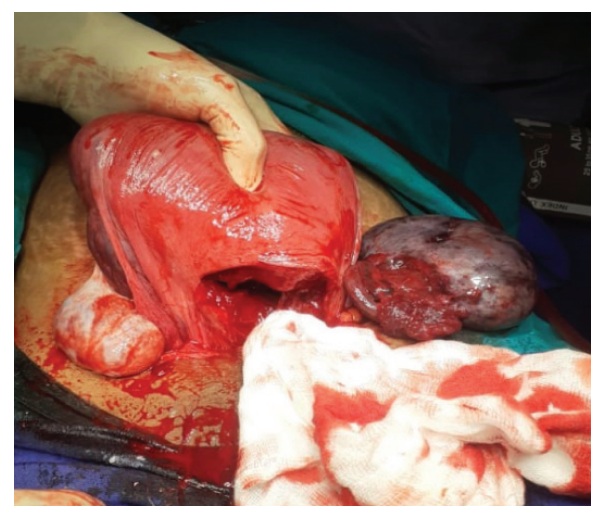

Table 1:Demographic characteristics of the patients

\begin{tabular}{|c|c|c|c|c|}
\hline \multicolumn{2}{|c|}{ Characteristics } & Number of cases & Percentage \% \\
\hline 1 & Age in years & $20-24$ & 15 & 46.87 \\
\hline & & $25-29$ & 14 & 43.75 \\
\hline & & $30-34$ & 3 & 9.37 \\
\hline 2 & Parity & Mean & 13 & 40.62 \\
\hline & & Primi & 19 & 59.37 \\
\hline 3 & Socioeconomic status & Multi & 15 & 46.87 \\
\hline & & Lower & 12 & 37.5 \\
\hline & & Middle & 18 & 56.25 \\
\hline 4 & Trimester at presentation & $\begin{array}{c}\text { First } \\
\text { (upto 12 weeks) }\end{array}$ & $\begin{array}{c}\text { Second } \\
\text { (13-28weeks) }\end{array}$ & $\begin{array}{c}\text { Third } \\
\text { (29-40weeks) }\end{array}$ \\
\hline & & $\begin{array}{c}\text { Mean gestational age in } \\
\text { weeks }\end{array}$ & 15.96 & 28.12 \\
\hline & & & 5 & 15.62 \\
\hline
\end{tabular}

Table 2:Indications for surgery of ovarian cysts

\begin{tabular}{|l|c|c|}
\hline Complications of ovarian cysts & Number of cases & Percentage \% \\
\hline Torsion & 3 & 9.37 \\
\hline Increase in size of the cyst & 4 & 12.5 \\
\hline Rupture & 0 & \\
\hline Hemorrhage & 0 & \\
\hline Hospitalisation due to pain & 0 & \\
\hline
\end{tabular}

Table 3: Obstetric outcome

\begin{tabular}{|l|c|c|}
\hline Complications of ovarian cysts & Number of cases & Percentage \% \\
\hline Vaginal delivery & 14 & 43.75 \\
\hline Cesarean section & 18 & 56.25 \\
\hline Miscarriage & 0 & \\
\hline
\end{tabular}




\section{Discussion}

Ovarian masses are diagnosed in $0.5-1 \%$ of pregnancies. In our study incidence is around $0.89 \%$. Goh W et al. in their study found that $1 \%$ of all pregnancies were diagnosed with an adnexal mass. ${ }^{8}$ Bernhard LM et al. in their study stated that $76 \%$ of adnexal cysts in pregnancy were simple ovarian cysts of $<5 \mathrm{~cm}$ in diameter. ${ }^{2}$ In our study $19(36.53 \%)$ of ovarian cysts were $<5 \mathrm{~cm}$ in size and were not included in our study.

In our study mean age at presentation was 25.18 years with maximum patients in the age group of $20-24$ years. Forty percent of them were primigravida and $59.37 \%$ were multigravida. This was similar to study conducted by Sheela et al. where $52.2 \%$ of cases were in the age group $20-24$ years and $43.5 \%$ primi gravida and $56.5 \%$ multigravida. ${ }^{9}$ Saba Musharaf et al. reported that at the time of presentation $57.5 \%$ were in first trimester, $32.5 \%$ in second and $10 \%$ in third trimester. ${ }^{10}$ In our study $18(56.25 \%)$ cases presented in first trimester, $9(28.12 \%)$ in second and $5(15.62 \%)$ in third trimester with mean gestational age at presentation being 15.96 weeks.

Bernhard LM et al. reported that 70 of 102 large or complex adnexal masses during pregnancy resolved spontaneously. ${ }^{2}$ In our study $25(78.12 \%)$ were conservatively managed, of which $18(56.25 \%)$ patients had spontaneous resolution. This is similar to a meta analyses conducted by Bascietto $\mathrm{F}$ et al. where in $53.8 \%$ of cases had spontaneous resolution of cyst. ${ }^{11}$ In our study about $7(21.87 \%)$ patients in whom the cyst was persistent throughout the antenatal period or was incidental finding during cesarean section, ovarian cystectomy was performed during cesarean section. Goh W et al. reported that with advent of ultrasound imaging in diagnosing rare malignant lesions, benign and asymptomatic masses can be allowed expectant management until delivery or postpartum and this expectant management of asymptomatic masses does not increase risk of adverse pregnancy outcomes. ${ }^{8}$

In our study $7(21.87 \%)$ underwent surgery, of which $4(12.5 \%)$ had elective laparotomy during second trimester due to increase in size of the ovarian cyst and $3(9.3 \%)$ had emergency surgery due to torsion of the ovarian cyst. Of the three one patient had laparoscopic cystectomy in first trimester, second patient had laparotomy and oophorectomy in second trimester and third patient with torsion presented at 36 weeks in whom caesarean section along with oophorectomy was performed. This is similar to the study conducted by Sheela SR et al. where the most common indication for surgery during pregnancy was increasing size of the cyst in $13 \%$ of cases and torsion in $4.3 \%$ of cases. ${ }^{9}$ Agarwal $\mathrm{P}$ et al. in their study reported that both laparoscopy and laparotomy had similar result with regard to obstetric outcome. ${ }^{5}$

Balinskaite $\mathrm{V}$ et al. in their study found that risk associated with non obstetric surgery was relatively low, confirming that surgical procedures during pregnancy are generally safe..$^{12}$ In our study $14(43.75 \%)$ of them had vagina delivery and $18(56.25 \%)$ had cesarean section at term. There were no miscarriages and $96.87 \%$ of patients had term deliveries similar to study conducted by Saba Musharaf et al. ${ }^{10}$ Koo et al. in their study reported that $42 \%$ of ovarian cysts during pregnancy were teratomas, $13 \%$ were cystadenomas, $6.5 \%$ endometriomas and $16.8 \%$ as functional cysts. ${ }^{13}$ But in our study out of 14 patients who underwent cystectomy $5(35.71 \%)$ were simple cyst, 5(35.71\%) were serous cystadenomas, $3(21.42 \%)$ mucinous cystadenomas and $1(7.14 \%)$ dermoid cyst.

\section{Conclusion}

Most of the ovarian cysts during pregnancy are benign, asymptomatic and resolve spontaneously. Optima management is conservative. Surgical management is for symptomatic patients. Both open surgery and laparoscopy can be performed in all trimesters with no adverse effect to the pregnancy outcome.

\section{REFERENCES}

1. Hermans RH, Fischer DC, van der Putten HW,et al. Adnexal masses in pregnancy. Onkologie.2003;26(2):167-172.

2. Bernhard LM, Klebba PK, Gray DL, et al. Predictors of persistence of adnexal masses in pregnancy. Obstet and Gynecol.1999;93(4):585-589.

3. Leiserowitz GS, Xing G, Cress R, et al. Adnexal masses in pregnancy: how often are they malignant? Gynecologic Oncology.2006;101(2):315-321.

4. Yen CF, Lin SL, Murk W,et al. Risk analysis of torsion and malignancy for adnexal masses during pregnancy. Fertility and Sterility.2009;91(5):1895-1902.

5. Aggarwal P, Kehoe S. Ovarian tumors in pregnancy: a literature review. European Journal of Obstetrics and Gynecology and Reproductive Biology. 2011;155(2):119124.

6. Schmeler KM, Mayo-smith WW, Peipert JF, et al. Adnexal masses in pregnancy: surgery compared with observation. Obststetrics and Gynecology.2005;105(5):1098-1103.

7. Baser E, Erkilinc S, Esin S, et al. Adnexal masses encountered during cesarean delivery. International Journal of Gynecology and Obstetrics. 2013;123(2):124126.

8. Goh W, Bohrer J, Zalud I. Management of the adnexal mass in pregnancy. Curr Opin Obstet Gynecol. 2014;26(2):49-53.

9. Sheela SR, Sreeramulu PN, Poonguzhali L, Arulselvi K. Obstetric outcome in pregnancy complicated by ovarian cysts. Int J Reprod Contracept Obstet Gynecol 2017;6(11):5051-5054. 
10. Saba Musharaf, Saba Malik, Mohd Abass, Fidah Mohamad. Adnexal Mass in Pregnancy: Obstetric Outcome and Management. Int J Med Res Prof. 2018; 4(6):82-84.

11. Bascietto F, Liberati M, Marrone L, et al. Outcome of fetal ovarian cysts diagnosed on prenatal ultrasound examination: systematic review and meta-analysis. Ultrasound Obstet Gynecol. 2017;50(1):20-31.

12. Balinskaite V, Bottle A, Sodhi V, Rivers A, et al. The Risk of Adverse Pregnancy Outcomes Following Nonobstetric Surgery During Pregnancy: Estimates From a Retrospective Cohort Study of 6.5 Million Pregnancies. Ann Surg. 2017;266(2):260-266.

13. Koo Y, Kim HJ, Lim K, et al. Laparotomy versus laparoscopy for the treatment of adnexal masses during pregnancy. Australian and New Zealand Journal of Obstestrics and Gynaecology. 2012;52(1):34-38.

How to cite this article : Singham G,Vivekananda A,Siripurapu S,Mohapatra I . Ovarian cysts in Pregnancy: Obstetric Outcome and Management. Perspectives in Medical Research 2021; 9 (1):69-73 DOI:10.47799/pimr.0901.14

Sources of Support: Nil, Conflict of interest: None declared 